\title{
High strain mechanical loading rapidly induces tendon apoptosis: an ex vivo rat tibialis anterior model
}

\author{
A Scott, K M Khan, J Heer, J L Cook, O Lian, V Duronio
}

Br J Sports Med 2005;39:e25 (http://www.bjsportmed.com/cgi/content/full/39/5/e25). doi: 10.1136/bjsm.2004.015164

\begin{abstract}
Background: The role of apoptosis, or programmed cell death, has only recently been explored in tendon.

Objective: To investigate the development of apoptosis after high strain loading of rat tendon.

Methods: The right tibialis anterior tendons of three rats were prepared for mechanical loading, and left tendons were prepared identically as non-loaded controls. Tendon was loaded with $20 \%$ strain for six hours using a $1 \mathrm{~Hz}$ longitudinal sine wave signal. The following were used to assess apoptosis: (a) a monoclonal mouse antibody (F7-26) to label single stranded DNA breaks; (b) a rabbit polyclonal antibody that specifically recognises the cleaved form of caspase-3.

Results: Light microscopy confirmed that the high strain protocol induced a stretch overload injury. Control tendons showed little or no staining with the F7-26 antibody, but the loaded tendons displayed numerous apoptotic cells. The percentage of apoptotic cells (20\%) in the loaded tendon was significantly greater than in the control tendon $(1 \%)(p=$ $0.000)$. The labelled cells colocalised with abnormal nuclear morphology, including nuclear fragmentation. The staining against cleaved caspase- 3 was positive in loaded tendons only, and localised both to nucleus and cytoplasm.

Conclusion: This experiment extends knowledge of human tendon apoptosis by showing that apoptosis can occur in response to short term, high strain mechanical loading. This is the first report of mechanical loading of intact tendon causing excessive apoptosis.
\end{abstract}

$\mathrm{T}$ endon disorders are an important cause of sports and work related impairment to quality of life. ${ }^{12}$ Historically, overuse tendon disorders were considered a classic "inflammatory" tendinitis, ${ }^{3}{ }^{4}$ but contemporary evidence suggests that other processes probably play a major role in the pathogenesis of painful tendon conditions. ${ }^{5-16}$ Apoptosis, or programmed cell death, contributes to homoeostasis in many adult tissues, and excessive apoptosis is associated with pathological conditions. ${ }^{6} 1718$ The role of apoptosis in the tendon, however, has only recently been explored..$^{17-20}$

Yuan $e t a l^{17}{ }^{18}$ were the first to report excessive apoptosis in tendon. In older patients (mean age $>60$ years) undergoing surgery for torn rotator cuff, the percentage of apoptotic cells $(34 \%)$ was significantly higher than in control tendon tissue (13\%). ${ }^{17} 18$ This important study also established that apoptosis was localised mainly to fibroblast-like cells. The authors encouraged further investigation of whether apoptosis contributes to tendon damage and predisposes to rupture, or occurs secondary to the rupture itself.

Sports related tendon apoptosis is probably associated with mechanical overuse, and several studies have examined the effect of mechanical straining of tendon cells in culture. ${ }^{20-26}$
Cell culture has many advantages for research, but results do not always mirror biology in vivo or ex vivo. To our knowledge, no study has investigated the development of apoptosis after loading of animal tendon tissue in a controlled fashion. Thus we provided a high strain load on the rat tibialis anterior in an attempt to induce tendon apoptosis.

\section{METHODS \\ Tendon tissue}

The rat tibialis anterior was selected because of its uniform width $(\sim 1.5 \mathrm{~mm})$ throughout its length $(\sim 10 \mathrm{~mm})$. Three mature male Long-Evans rats were killed with carbon dioxide. As significant changes (see below) were already present after examination of the third loaded specimen, we report on three experimental and three control specimens only. The six tibialis anterior tendons were exposed, and the muscle belly of tibialis anterior was separated from surrounding structures so the tendon could be dissected free without direct contact. The tendons were rinsed three times in sterile saline and maintained overnight in Dulbecco's modified eagle's medium containing $10 \%$ fetal bovine serum and $100 \mathrm{U} / \mathrm{ml}$ penicillin, streptomycin, glutamine (DMEM) at $37^{\circ} \mathrm{C}$ and buffered with $25 \mathrm{mM}$ Hepes buffer. After the overnight incubation, the paratenon could easily be removed by gently pulling with tweezers.

\section{Mechanical loading protocol}

The tendon proper was clamped at either end with custom built, toothed aluminium grips and submerged in a heated $\left(37^{\circ} \mathrm{C}\right)$ organ bath containing pre-warmed DMEM which was refreshed every 15 minutes. One grip was fixed to a stationary clamp, and the other grip (muscle end) was attached to a moveable arm placed so as to eliminate tendon slack. This yielded a grip to grip length of $6.4(0.4) \mathrm{mm}$. The moveable arm was programmed to oscillate in a longitudinal sine wave at $1 \mathrm{~Hz}$, and the strain was gradually increased to $20 \%$ of the grip to grip length. This supraphysiological load was maintained for six hours. The right tendon from each of three animals was loaded, and the left served as control and was treated identically except that it was not subjected to any strain.

\section{Light microscopy examination}

After the six hour loading protocol, tendons were cut from the grips with a sterile scalpel, rinsed with sterile phosphate buffered saline (PBS) and maintained in DMEM in a $5 \% \mathrm{CO}_{2}$ tissue culture incubator for 24 hours to allow a substantial number of cells to commit to apoptosis. Next, the tissue was fixed in fresh, cold 4\% paraformaldehyde for 24 hours, embedded in paraffin and sectioned at $5 \mu \mathrm{m}$. Haematoxylin and eosin sections were examined on a Zeiss Axioplan using objectives up to $63 \times$ magnification, and digital images were obtained. 


\section{Detection of apoptosis}

Two immunohistochemical methods were used to examine apoptosis in loaded and control tendon tissue. The first used a monocolonal mouse antibody (F7-26) that labels single stranded DNA breaks (Chemicon, Temecula, California, USA). Tissue sections were deparaffinised in xylene, then washed sequentially in 100\%, 95\%, and 70\% ethanol and PBS. Slides were incubated in PBS containing $0.2 \mathrm{mg} / \mathrm{ml}$ saponin and $20 \mu \mathrm{g} / \mathrm{ml}$ Proteinase $\mathrm{K}$ at room temperature for $20 \mathrm{~min}$ utes to expose the antigen. They were then washed in distilled water and transferred to $50 \%$ formamide ( $\mathrm{v} / \mathrm{v}$ distilled water) for 20 minutes to denature the DNA. After blocking with $3 \%$ non-fat dry milk, the sections were incubated with F7-26 $(10 \mu \mathrm{g} / \mathrm{ml})$ for 30 minutes at room temperature, then with biotinylated goat anti-mouse IgM (1:1000; Dako) for 30 minutes. Finally, the sections were incubated with avidin-fluorescein isothiocyanate (Sigma, St Louis, Missouri, USA), counterstained with propidium iodide, and mounted with gel mount (Vector, Burlingame, California, USA).

The second method used a rabbit polyclonal antibody that specifically recognises the cleaved form of caspase-3 (Cell Signaling Technology, Beverley, California, USA). The sections were cleared as described above, then permeabilised with methanol for 10 minutes. Endogenous peroxidases were quenched with $3 \%$ hydrogen peroxide. The sections were incubated with the primary antibody $(1: 50)$ overnight at $4{ }^{\circ} \mathrm{C}$, then visualised using an avidin/biotin complex kit with diaminobenzidine as the substrate (Vector) and Harris' haematoxylin as the counterstain. For both antibodies, one section per slide was incubated with mouse or rabbit serum (rather than the primary antibody) that eliminated the specific antibody staining. To quantify apoptosis from the sections stained with monoclonal mouse antibody (F27-6), one investigator (AS) counted cells from non-overlapping fields in both loaded and control tendon while remaining blinded to the group.

\section{Statistical analysis}

All values are expressed as mean (SD). The distribution of cell counts approximated normality. Thus statistical comparison of cell counts in loaded and unloaded tendon was performed using unpaired Student's $t$ tests. The confidence limit was predetermined at an $\alpha$ level of 0.05 .

\section{RESULTS}

Description of tendon tissue and macroscopic change At the end of the six hour protocol, the loaded tendons had lost their normal, tightly packed appearance. The ends immediately adjacent to the grips appeared grossly frayed. They were ignored in the analysis to eliminate the possibility of examining tendon that had been directly injured by the grips.

\section{Light microscopy appearance}

The haematoxylin and eosin sections of control tendons showed the typical, tightly packed arrangement of collagen fibres. Most tenocytes were spindle shaped, with sparse cytoplasm, arranged in longitudinal arrays. Superficial regions of the tendon occasionally displayed rounded tenocytes, some of which were located in lacunae. The rat tibialis anterior tendon did not display any vessels or nerves. The haematoxylin and eosin sections of the loaded tendons confirmed that the high strain protocol had induced a stretch overload injury (fig $1 \mathrm{~A}, \mathrm{~B}$ ). There was evidence of injury to both cells (tenocytes) and collagen fibres. Collagen fibres
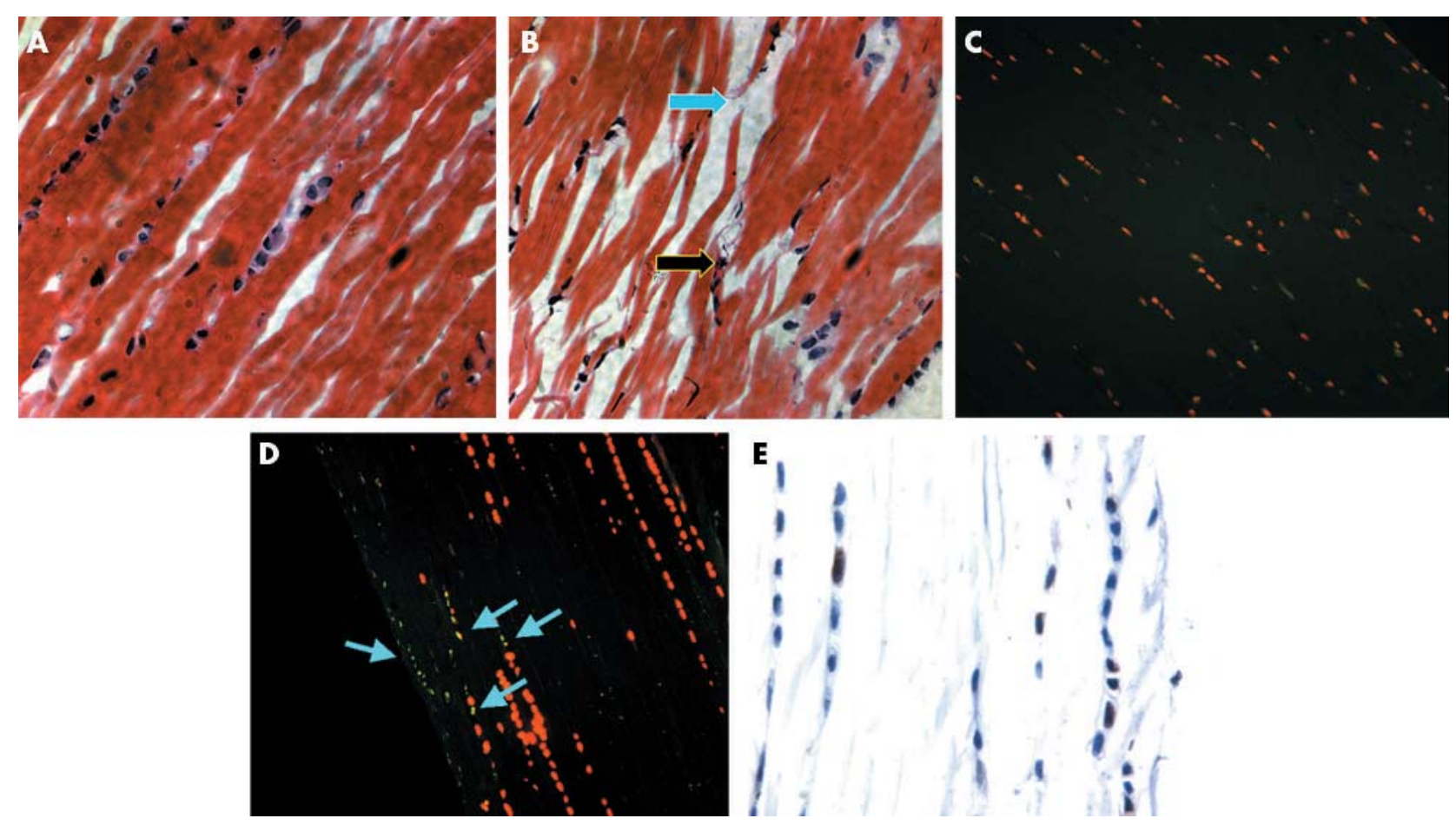

Figure 1 Cell death resulting from supraphysiological loading (20\% strain for six hours) of the rat tibialis anterior tendon. Haematoxylin and eosin stain after the loading experiment shows the characteristic well organised features of normal tendon (A, control tendon) and the disarray of tendon subjected to loading (B). In the loaded tendon, the blue arrow points to disrupted collagen fibres and the black arrow to nuclear fragmentation, a hallmark of apoptosis. Monoclonal mouse antibody (F27-6) staining for single stranded DNA breaks revealed virtually no apoptotic cells in the control tendon (C) but cells positive for apoptosis in the loaded specimen (D). Finally, a rabbit polyclonal antibody specifically recognising the cleaved end of caspase-3 revealed positive staining localised to the nucleus in loaded tendon (E). 
What is already known on this topic

Apoptosis is a non-inflammatory, often physiological, process of cell suicide which can be distinguished from necrosis, a process that is associated with inflammation. Cells of injured rotator cuff tendons have much higher rates of apoptosis than cells of uninjured tendons. Whether this is a cause or an effect of tendinopathy remains unknown.

displayed longitudinal separation as well as transverse tears, with fraying of fascicles. There were three main types of tenocyte abnormalities observed in loaded tendons. The first were regions of complete absence of tenocytes. This was reflected in the lower number of tenocytes observed per viewing field. In the second abnormality, injured tenocytes appeared larger than normal, with prominent cytoplasm and a more polygonal or rounded shape. In the third abnormality, tenocytes appeared shrunken, with fragmented nuclei consistent with apoptosis.

\section{Apoptosis assays}

Control tendons showed little or no staining (zero or single positive cells per section) with the F7-26 antibody. In contrast, the loaded tendons displayed numerous apoptotic cells (fig 1C,D). The labelled cells typically colocalised with abnormal nuclear morphology, including nuclear fragmentation or relative loss of nuclear staining. The staining against cleaved caspase- 3 showed a similar pattern, with positively labelled cells only detected in the loaded tendons, but the staining localised both to nucleus and cytoplasm (fig $\mathrm{IE}$ ). Identical results were also found with tendons that were quick frozen rather than paraffin fixed, and fixed in acetone rather than paraformaldehyde.

Apoptosis quantification revealed that the mean (SD) number of apoptotic cells per field in loaded tendon was 21 (4) whereas in control tendon it was 2 (2) apoptotic cells per field $(\mathrm{p}=0.000)$. There was no difference in the number of total cells per field in the two conditions (loaded $=115$ (32) compared with unloaded $=120(25))$. Thus, in the loaded and control tendon, apoptotic cells comprised $20.2 \%$ and $1.3 \%$ of the cells respectively.

\section{DISCUSSION}

Our experiment extends the pivotal report of Yuan and colleagues $^{18}$ on tendon apoptosis in long term rotator cuff injury (complete tear of supraspinatus) by showing that apoptosis can occur in response to short term high strain mechanical loading. To our knowledge, this is the first report of mechanical loading of intact tendon-that is, not cell culture-causing excessive apoptosis. However, whether tendon cell apoptosis is involved in the regulation of human overuse tendon pathology, or only acts as a normal physiological response to high strain mechanical load, remains to be studied.

A strength of this study was the use of two distinct methods to demonstrate apoptosis. We used highly specific antibodies recognising fragmented DNA and cleaved caspase3, allowing us to conclude that apoptosis had occurred. This immunohistochemical approach is more specific than other viability assays, such as in situ terminal transferase mediated nick end labelling (TUNEL), that potentially label both apoptotic and necrotic cells. We found that $20 \%$ of cells in the loaded tendon were apoptotic. This is comparable to the published report that $34 \%$ of cells in chronic rotator cuff tears were apoptotic. $^{18}$
What this study adds

This is the first report of mechanical loading of intact tendon-that is, not cell culture-causing excessive apoptosis. Highly specific antibodies recognising fragmented DNA and cleaved caspase-3 demonstrated that apoptosis had occurred. This immunohistochemical approach is more specific than other viability assays, such as TUNEL, that potentially label both apoptotic and necrotic cells.

Further studies should address whether variations in the mechanical loading protocol influence the process of tendon apoptosis. Whether response depends on load strain itself, frequency, or strain rate warrants investigation. Animal studies that used bone as the tissue of interest, for example, showed that strain rate has a greater influence on osteogenesis than does number of strain cycles. ${ }^{27-29}$ Other bone studies have emphasised the importance of rest periods within quanta of mechanical loading. ${ }^{30}{ }^{31}$ Whether there is such a relation between mechanical loading and tendon apoptosis needs study, and animal models can permit this type of investigation. ${ }^{32}$

Tendon apoptosis is not a simple, linear pathway. There are two major apoptotic pathways ${ }^{33}$ (mitochondrial or intrinsic, and death receptor or extrinsic), and research is needed into how these pathways are associated with the apoptotic response we have shown in this experiment. This is likely to be an important area of investigation as discoveries in cell signalling continue.

Once the effect of mechanical loading on tendon apoptosis has been better characterised, future research will investigate whether growth factors and cytokines can influence (either retard or accelerate) the apoptotic processes. ${ }^{24}{ }^{34}$ Both the characterisation of the signalling pathways and the research into apoptosis modulators have the potential to contribute to targeted treatments for tendinopathy.

This small experiment had several limitations that can be overcome in future studies. The mechanical loading device that we used did not allow us to precisely measure the strain being seen within the tendon itself. We propose that future studies use load devices such as the Instron and implant strain gauges within tendon to overcome these limitations. The strains that are associated with the development of tendon injuries, in vivo, are much smaller than those used in this study, and future studies should test the dimensions of strain that promote, or protect against, development of apoptosis.

As in any animal study, the question of the model and its relevance to human tissue arises..$^{35}$ The rat tibialis anterior is readily available and large enough to allow mechanical loading ex vivo. The overuse rat tendon model of Soslowsky and coworkers ${ }^{36}{ }^{37}$ duplicates many features of human pathology.

In summary, the present study presents novel findings, showing that it is possible to induce tendon apoptosis with supraphysiological mechanical loading. This model for ex vivo study of tendon apoptosis warrants further investigation.
Authors' affiliations
A Scott, K M Khan, V Duronio, Department of Family Practice \& School of Human Kinetics, University of British Columbia, Vancouver, Canada J Heer, University of Melbourne, Australia
J L Cook, La Trobe University, Melbourne, Australia O Lian, Oslo Sports Trauma Research Centre, Oslo, Norway
Competing interests: none declared 
Correspondence to: Assistant Professor Khan, Department of Family Practice \& School of Human Kinetics, University of British Columbia, Suite 211, 2150 Western Parkway, Vancouver V6T 1V6, Canada; kkhan@interchange.ubc.ca

Accepted 23 November 2004

\section{REFERENCES}

1 Maffulli N, Wong J, Almekinders LC. Types and epidemiology of tendinopathy. Clin Sports Med 2003;22:675-92.

2 Khan KM, Maffulli N. Tendinopathy: an Achilles' heel for athletes and clinicians. [Lead editorial]. Clin J Sport Med 1998;8:151-4.

3 Scott A, Khan KM, Roberts CR, et al. What do we mean by the term "inflammation"? A contemporary basic science update for sports medicine. Br J Sports Med 2004;38:372-80.

4 Scott A, Khan KM, Cook JL, et al. What is "inflammation"? Are we ready to move beyond Celsus? Br J Sports Med 2004;38:248-9.

5 Khan KM, Cook J, Kannus P, et al. Time to abandon the 'tendinitis' myth. BMJ 2002;324:626-7.

6 Murrell GA. Understanding tendinopathies. Br J Sports Med 2002;36:392-3.

7 Kjaer M. Role of extracellular matrix in adaptation of tendon and skeletal muscle to mechanical loading. Physiol Rev 2004;84:649-98.

8 Bestwick CS, Maffulli N. Reactive oxygen species and tendon problems: review and hypothesis. Sports Med Arthrosc Rev 2000;8:6-16.

9 Alfredson $\mathrm{H}$. Chronic tendon pain: implications for treatment: an update. Curr Drug Targets 2004;5:407-10.

10 Ohberg L, Alfredson $\mathrm{H}$. Effects on neovascularisation behind the good results with eccentric training in chronic mid-portion Achilles tendinosis? Knee Surg Sports Traumatol Arthrosc 2004; 12:465-70.

11 Ljung BO, Alfredson H, Forsgren S. Neurokinin 1-receptors and sensory neuropeptides in tendon insertions at the medial and lateral epicondyles of the humerus. Studies on tennis elbow and medial epicondylalgia. J Orthop Res 2004;22:321-7.

12 Ackermann PW, Finn A, Ahmed M. Sensory neuropeptidergic pattern in tendon, ligament and joint capsule. A study in the rat. Neuroreport 1999;10:2055-60.

13 Ackermann PW, Spetea M, Nylander I, et al. An opioid system in connective tissue: a study of achilles tendon in the rat. $J$ Histochem Cytochem 2001:49:1387-95.

14 Ackermann PW, Li J, Finn A, et al. Autonomic innervation of tendons, ligaments and joint capsules. A morphologic and quantitative study in the rat. $J$ Orthop Res 2001;19:372-8.

15 Ackermann PW, Li J, Lundeberg T, et al. Neuronal plasticity in relation to nociception and healing of rat achilles tendon. J Orthop Res 2003;21:432-41.

16 Ackermann PW, Ahmed M, Kreicbergs A. Early nerve regeneration after achilles tendon rupture--a prerequisite for healing? A study in the rat. J Orthop Res 2002;20:849-56.

17 Yuan J, Wang MX, Murrell GA. Cell death and tendinopathy. Clin Sports Med 2003;22:693-701.

18 Yuan J, Murrell GA, Wei $A Q$, et al. Apoptosis in rotator cuff tendonopathy. $J$ Orthop Res 2002;20:1372-9.
19 Yuan J, Murrell GA, Trickett A, et al. Involvement of cyłochrome c release and caspase-3 activation in the oxidative stress-induced apoptosis in human tendon fibroblasts. Biochim Biophys Acta 2003;1641:35-41.

20 Arnoczky SP, Tian T, Lavagnino M, et al. Activation of stress-activated protein kinases (SAPK) in tendon cells following cyclic strain: the effects of strain frequency, strain magnitude, and cytosolic calcium. J Orthop Res 2002;20:947-52.

21 Banes AJ, Donlon K, Link GW, et al. Cell populations of tendons: a simplified method for isolation of synovial cells and internal fibroblasts. Confirmation of biologic properties. J Orthop Res 1988:6:83-94.

22 Banes AJ, Hu P, Xiao H, et al. Tendon cells of the epitenon and internal tendon compartment communicate mechanical signals through gap junctions and respond differentially to mechanical load and growth factors. In:Gordon SL, Blair SJ, Fine L, editors. Repetitive motion disorders of the upper extremity. Rosemount: American Academy of Orthopedic Surgeons, 1995:231-45.

23 Banes AJ, Tsuzaki M, Yamamoto J, et al. Mechanoreception at the cellular level: the detection, interpretation, and diversity of responses to mechanical signals. Biochem Cell Biol 1995:73:349-65.

24 Banes AJ, Tsuzaki M, Hu P, et al. PDGF-BB, IGF-I and mechanical load stimulate DNA synthesis in avian tendon fibroblasts in vitro. J Biomech 1995;28:1505-13

25 Banes AJ, Weinhold P, Yang X, et al. Gap junctions regulate responses of tendon cells ex vivo to mechanical loading. Clin Orthop 1999;367(suppl):S356-70.

26 Banes AJ, Horesovsky G, Larson C, et al. Mechanical load stimulates expression of novel genes in vivo and in vitro in avian flexor tendon cells. Osteoarthritis Cartilage 1999:71:141-53.

27 Umemura $Y$, Ishiko $T$, Tsujimoto $H$, et al. The effects of jump training on bone hypertrophy in young and old rats. Int I Sports Med 1995;16:364-7.

28 Umemura Y, Ishhiko T, Yamauchi T, et al. Five jumps per day increase bone mass and breaking force in rats. J Bone Miner Res 1997;12:1480-5.

29 Turner CH, Owan I, Takano Y. Mechanotransduction in bone: role of strain rate. Am J Physiol 1995;269:E438-42.

30 Gross TS, Poliachik SL, Ausk BJ, et al. Why rest stimulates bone formation: a hypothesis based on complex adaptive phenomenon. Exerc Sport Sci Rev 2004;32:9-13.

31 Srinivasan S, Weimer DA, Agans SC, et al. Low-magnitude mechanical loading becomes osteogenic when rest is inserted between each load cycle. $J$ Bone Miner Res 2002;17:1613-20.

32 Thorndike EA, Turner AS. In search of an animal model for postmenopausal diseases. Front Biosci 1998:3:c17-26.

33 Debatin KM. Apoptosis pathways in cancer and cancer therapy. Cancer Immunol Immunother 2004;53:153-9.

34 Molloy $T$, Wang Y, Murrell G. The roles of growth factors in tendon and ligament healing. Sports Med 2003;33:381-94.

35 Soslowsky L, Carpenter JE, DeBano CM, et al. Development and use of an animal model for investigations on rotator cuff disease. J Shoulder Elbow Surg 1996;5:383-92.

36 Carpenter JE, Thomopoulos S, Soslowsky $\sqcup$. Animal models of tendon and ligament injuries for tissue engineering applications. Clin Orthop 1999;367(suppl):S296-311.

37 Soslowsky L, Thomopoulos S, Tun S, et al. Neer Award 1999. Overuse activity injures the supraspinatus tendon in an animal model: a histologic and biomechanical study, J Shoulder Elbow Surg 2000;9:79-84. 